\title{
Detecting discontinuities over triangular meshes using multiwavelets
}

\author{
Mathea J. Vuik and Jennifer K. Ryan
}

\begin{abstract}
It is well known that solutions to nonlinear hyperbolic PDEs develop discontinuities in time. The generation of spurious oscillations in such regions can be prevented by applying a limiter in the troubled zones. In earlier work, we constructed a multiwavelet troubled-cell indicator for one and (tensor-product) two dimensions (SIAM J. Sci. Comput. 38(1):A84-A104, 2016). In this paper, we investigate multiwavelet troubled-cell indicators on structured triangular meshes. One indicator uses a problem-dependent parameter; the other indicator is combined with outlier detection.
\end{abstract}

\section{Introduction}

It is well known that solutions to nonlinear hyperbolic PDEs develop discontinuities in time. The generation of spurious oscillations in such regions can be prevented by applying a limiter in the troubled zones. In $[18,19,20]$, two different multiwavelet troubled-cell indicators were introduced, one based on a parameter, the other combined with outlier detection. In these papers, we focused on one-dimensional and tensor-product two-dimensional meshes. In this paper, the use of multiwavelets on triangular meshes is investigated $[17,21]$. We again consider two approaches to troubled-cell indication: one based on a parameter, the other combined with outlier detection. We demonstrate the performance of the indicators on a test problem based on the two-dimensional linear advection equation, using the vertex-based limiter in the identified troubled cells [10].

Mathea J. Vuik

VORtech, Post Box 260, 2600AG Delft, The Netherlands, e-mail: thea.vuik@ vortech.nl

Jennifer K. Ryan

School of Mathematics, University of East Anglia, Norwich NR4 7TJ, United Kingdom, e-mail: Jennifer.Ryan@uea.ac.uk 
The outline of this paper is as follows: in Section 2, the triangular mesh is defined, and information about barycentric coordinates is given. The multiresolution analysis is described in Section 3. The multiwavelet troubled-cell indicators are defined in Section 4. Preliminary results are shown in Section 5, and some concluding remarks are given in Section 6.

\section{Structured triangular mesh and barycentric coordinates}

In this section, the definition of a structured triangular mesh on a rectangular domain $\Omega \in \mathbb{R}^{2}$ is given, following the notation in $[5,17]$. To compute the multiwavelet decomposition at a later time, the relation between the mesh on the finest level $n$ and level $n-1$ is explained. Furthermore, several properties of the barycentric coordinate system are given.

Definition 1. Let $i$ and $j$ be space indices in the $x$ - and $y$-direction, respectively, and let $M$ account for the orientation of the triangle: $M=1$ corresponds to triangles with the right angle located in the bottom-left corner, $M=2$ belongs to the triangles with right angles in the upper-right corner. The uniform triangulation of a rectangular domain $\Omega \in \mathbb{R}^{2}$ on level $n$ consists of $2^{2 n+1}$ elements, and is expressed as

$$
\mathscr{T}^{n}=\left\{T_{(i, j, M)}^{n}\right\}_{i, j=0, \ldots, 2^{n}-1}^{M=1,2}=\left\{T_{\lambda}^{n}\right\}_{\lambda},
$$

with $\lambda=(i, j, M), i, j=0, \ldots, 2^{n}-1, M=1,2$.

The triangulation on level $n-1$ is obtained by uniting four triangles on level $n$ :

$$
\begin{aligned}
T_{(i, j, 1)}^{n-1} & =T_{(2 i, 2 j, 2)}^{n} \cup T_{(2 i, 2 j, 1)}^{n} \cup T_{(2 i+1,2 j, 1)}^{n}, \cup T_{(2 i, 2 j+1,1)}^{n}, \\
T_{(i, j, 2)}^{n-1} & =T_{(2 i+1,2 j+1,1)}^{n} \cup T_{(2 i+1,2 j+1,2)}^{n} \cup T_{(2 i, 2 j+1,2)}^{n} \cup T_{(2 i+1,2 j, 2)}^{n},
\end{aligned}
$$

$i, j=0, \ldots, 2^{n-1}-1$, see Figure 1 .

Points inside a triangle are efficiently expressed using barycentric coordinates.

Definition 2. Let triangle $T$ be defined by its vertices $\mathbf{P}_{i}=\left(x_{i}, y_{i}\right)^{\top}, i=1,2,3$. Every point $\mathbf{P}=(x, y)^{\top}$ can be expressed in terms of the barycentric coordinates $\boldsymbol{\tau}=\left(\tau_{1}, \tau_{2}, \tau_{3}\right)^{\top}$ with respect to triangle $T$ as follows: $\mathbf{P}=\left(\mathbf{P}_{1} \mathbf{P}_{2} \mathbf{P}_{3}\right) \boldsymbol{\tau}$.

The barycentric coordinates are uniquely given by requiring $|\tau|=\tau_{1}+\tau_{2}+\tau_{3}=1$. If $\mathbf{P}$ is located inside $T$, then $\tau_{i} \geq 0, i=1,2,3$.

Integrals on a triangle can be transformed to barycentric coordinates as follows:

$$
\iint_{T} f(x, y) d x d y=2|T| \int_{0}^{1} \int_{0}^{1-\tau_{1}} f\left(x\left(\tau_{1}, \tau_{2}\right), y\left(\tau_{1}, \tau_{2}\right)\right) d \tau_{2} d \tau_{1},
$$

where $|T|$ is the area of $T[17,21]$.

The transformation from original coordinates to barycentric coordinates equals 


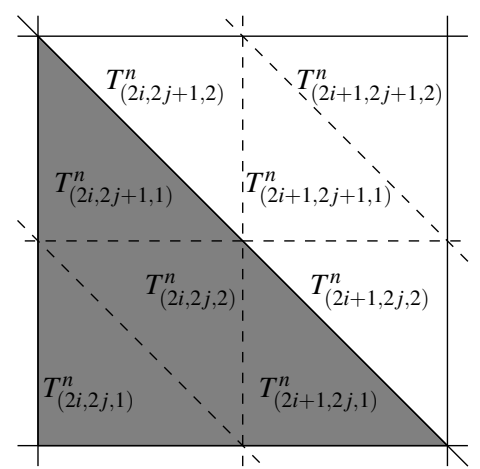

Fig. 1 Triangulation $\mathscr{T}^{n}$ of a rectangular domain $\Omega \in \mathbb{R}^{2}$. Solid lines correspond to the elements $T_{(i, j, 1)}^{n-1}$ and $T_{(i, j, 2)}^{n-1}$ on level $n-1$.

$$
\left(\begin{array}{l}
\tau_{1} \\
\tau_{2} \\
\tau_{3}
\end{array}\right)=\left(\begin{array}{ccc}
x_{1} & x_{2} & x_{3} \\
y_{1} & y_{2} & y_{3} \\
1 & 1 & 1
\end{array}\right)^{-1}\left(\begin{array}{l}
x \\
y \\
1
\end{array}\right)
$$

With this expression, it is possible to relate the barycentric coordinates on different triangles which will be necessary when discussing multiwavelets. If $\mathbf{P}$ has barycentric coordinates $\tau$ relative to triangle $T$ (defined by $\left\{\left(x_{i}, y_{i}\right), i=1,2,3\right\}$ ), then the barycentric coordinates $\tau^{\prime}$ with respect to $T^{\prime}$ (defined by $\left\{\left(x_{i}^{\prime}, y_{i}^{\prime}\right), i=1,2,3\right\}$ ) can be calculated using $\tau^{\prime}=M_{T \rightarrow T^{\prime}} \tau$, where

$$
M_{T \rightarrow T^{\prime}}=\left(\begin{array}{ccc}
x_{1}^{\prime} & x_{2}^{\prime} & x_{3}^{\prime} \\
y_{1}^{\prime} & y_{2}^{\prime} & y_{3}^{\prime} \\
1 & 1 & 1
\end{array}\right)^{-1}\left(\begin{array}{ccc}
x_{1} & x_{2} & x_{3} \\
y_{1} & y_{2} & y_{3} \\
1 & 1 & 1
\end{array}\right) .
$$

The right matrix transforms $\boldsymbol{\tau}$ to $\mathbf{P}$, and the left matrix computes $\boldsymbol{\tau}^{\prime}$ from $\mathbf{P}[17,21]$.

Finally, the midpoint subdivision of a triangle $T_{\lambda}^{n-1}=T_{\lambda_{0}}^{n} \cup T_{\lambda_{1}}^{n} \cup T_{\lambda_{2}}^{n} \cup T_{\lambda_{3}}^{n}$ can easily be described in barycentric coordinates, see Figure $2[17,21]$.

\section{Multiresolution analysis}

In this section, the multiresolution analysis for a triangular mesh is presented, together with the formulae for multiwavelet decomposition [17]. For the reconstruction procedure, we refer to [17]. The scaling functions and multiwavelets are constructed for the so-called base triangle, $T_{B}$, which has vertices $\mathbf{P}_{1}=(0,0)$, $\mathbf{P}_{2}=(1,0)$, and $\mathbf{P}_{3}=(0,1)$, and subdivision $T_{B}=T_{0} \cup T_{1} \cup T_{2} \cup T_{3}$ (numbering similar to Figure 2). The extension to general triangles is given as well. 


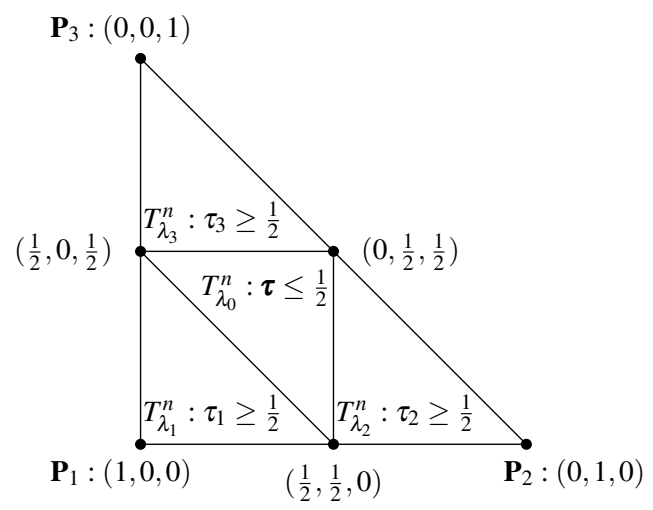

Fig. 2 Midpoint subdivision of triangle $T_{\lambda}^{n-1}$. Coordinates are in barycentric form $\left(\tau_{1}, \tau_{2}, \tau_{3}\right)$.

\subsection{Scaling-function space}

In this section, the orthonormal scaling-function basis is constructed for the base triangle, using barycentric coordinates [21]. The scaling-function space on $T_{B}$ is defined as $V^{k+1}\left(T_{B}\right)=\mathbb{P}^{k}\left(T_{B}\right)$, which means that the space is spanned by polynomials of total degree less than or equal to $k$ on $T_{B}$. The standard monomial basis for $V^{k+1}\left(T_{B}\right)$ consists of $N_{k}$ functions $\left\{1, x, y, x^{2}, x y, y^{2}, \ldots\right\}$. For the base triangle, the coordinates $(x, y)$ transform to

$$
\begin{aligned}
& x=\tau_{1} x_{1}+\tau_{2} x_{2}+\tau_{3} x_{3}=\tau_{2}, \\
& y=\tau_{1} y_{1}+\tau_{2} y_{2}+\tau_{3} y_{3}=\tau_{3}=1-\tau_{1}-\tau_{2}
\end{aligned}
$$

in barycentric coordinates. This means that the monomial basis is equivalent to the set $\left\{1, \tau_{2}, 1-\tau_{1}-\tau_{2}, \tau_{2}^{2},\left(1-\tau_{1}-\tau_{2}\right) \tau_{2},\left(1-\tau_{1}-\tau_{2}\right)^{2}, \ldots\right\}$ in the barycentric coordinate system. Orthonormality of this basis is achieved by the application of the Gram-Schmidt procedure with respect to the inner product

$$
\langle f, g\rangle=\int_{0}^{1} \int_{0}^{1-\tau_{1}} f\left(\tau_{1}, \tau_{2}\right) g\left(\tau_{1}, \tau_{2}\right) d \tau_{2} d \tau_{1},
$$

together with normalization. This results in the orthonormal scaling functions $\phi_{\ell, T_{B}}$, $\ell=1, \ldots, N_{k}$. The first six functions (corresponding to $k \leq 2$ ) are given in [17, 21].

The scaling-function space on level $n$ is defined as the space of piecewise polynomials of total degree less than or equal to $k$ on every triangle $T_{\lambda}^{n} \in \mathscr{T}^{n}$ :

$$
V_{n}^{k+1}=\left\{f: f \in \mathbb{P}^{k}\left(T_{\lambda}^{n}\right), \quad \forall T_{\lambda}^{n} \in \mathscr{T}^{n}\right\} .
$$

The orthonormal basis for $V_{n}^{k+1}$ can be found by substituting the correct barycentric coordinates (translation) and scaling the functions $\phi_{\ell, T_{B}}$ [21]. Let $\tau$ be the barycentric coordinates with respect to $T_{B}$, and let $\tau^{\prime}$ be the corresponding barycentric coor- 
dinates with respect to $T_{\lambda}^{n} \in \mathscr{T}^{n}$. The space $V_{n}^{k+1}$ is spanned by $2^{2 n+1} \cdot N_{k}$ functions that are obtained from $\phi_{\ell, T_{B}}$ using

$$
\phi_{\ell \lambda}^{n}\left(\tau_{1}^{\prime}, \tau_{2}^{\prime}, \tau_{3}^{\prime}\right)=\sqrt{\frac{1}{2\left|T_{\lambda}^{n}\right|}} \phi_{\ell, T_{B}}\left(\tau_{1}, \tau_{2}, \tau_{3}\right) .
$$
by

The orthogonal projection of an arbitrary function $f \in L^{2}(\Omega)$ onto $V_{n}^{k+1}$ is given

$$
P_{n}^{k+1} f(\mathbf{x})=\sum_{T_{\lambda}^{n} \in \mathscr{T}^{n}} \sum_{\ell=1}^{N_{k}} s_{\ell \lambda}^{n} \phi_{\ell \lambda}^{n}(\boldsymbol{\tau}),
$$

which is the single-scale decomposition of $f$ on level $n$. The scaling-function coefficients are given by $s_{\ell \lambda}^{n}=\left\langle f, \phi_{\ell \lambda}^{n}\right\rangle$. Note that if $f \in V_{n}^{k+1}$, then $P_{n}^{k+1} f=f$.

\subsection{Nodal DG approximation and scaling-function expansion}

Although it is possible to use modal DG based on a PKD-polynomial basis on triangular meshes [12], it is more convenient to use the nodal form of the DG method for this mesh type $[2,3,8]$.

The DG approximation space, $V_{h}$, is equal to the scaling-function space on level $n$ (equation (3)). This means that it is possible to express the nodal DG approximation as a scaling-function approximation in level $n$. Since $u_{h} \in V_{h}=V_{n}^{k+1}$, we know that $u_{h}=P_{n}^{k+1} u_{h}$. Therefore, we can write the global nodal DG approximation as

$$
u_{h}(\mathbf{x})=\sum_{T_{\lambda}^{n} \in \mathscr{T}^{n}} \sum_{i=1}^{N_{k}} u_{h}\left(\mathbf{x}^{i}\right) \ell_{i}(\mathbf{x})=\sum_{T_{\lambda}^{n} \in \mathscr{T}^{n}} \sum_{\ell=1}^{N_{k}} s_{\ell \lambda}^{n} \phi_{\ell \lambda}^{n}(\boldsymbol{\tau}) .
$$

Knowing the values $u_{h}\left(\mathbf{x}^{i}\right)$, we can efficiently compute the scaling-function coefficients by a matrix-vector multiplication. Define the vectors $\mathbf{s}_{\lambda}^{n}=\left(s_{1 \lambda}^{n}, \ldots, s_{N_{k} \lambda}^{n}\right)^{\top}$, $\mathbf{u}_{h}=\left(u_{h}\left(\mathbf{x}^{1}\right), \ldots, u_{h}\left(\mathbf{x}^{N_{k}}\right)\right)^{\top}$, and a Vandermonde matrix by $V_{m i}=\phi_{i \lambda}^{n}\left(\boldsymbol{\tau}\left(\mathbf{x}^{m}\right)\right)$, then $\mathbf{V} \mathbf{s}_{\lambda}^{n}=\mathbf{u}_{h}$ and $\mathbf{V}^{-1} \mathbf{u}_{h}=\mathbf{s}_{\lambda}^{n}$.

This procedure is very similar to the transformation from nodal to modal DG. This is because the scaling-function basis for $V_{n}^{k+1}$ is closely related to the modal DG basis, which is given by the so-called PKD polynomials [4, 9]. The difference between both bases is the reference triangle that is used [8].

\subsection{Multiwavelets}

In addition to the scaling-function space, the multiwavelet space should be defined. This is done by computing the orthogonal complement: $V_{n-1}^{k+1} \oplus W_{n-1}^{k+1}=V_{n}^{k+1}$, such 
that $W_{n-1}^{k+1} \perp V_{n-1}^{k+1}, W_{n-1}^{k+1} \subset V_{n}^{k+1}$. In Algorithm 6.1 in [17], the procedure to compute the multiwavelets for the base triangle is given, in a manner very similar to Alpert's construction in one dimension $[1,21]$. The execution of this algorithm leads to the multiwavelets as provided in [15].

Similar to equation (4), the multiwavelets on triangle $T_{\lambda}^{n} \in \mathscr{T}^{n}$ are equal to

$$
\psi_{\ell \lambda}^{m, n}\left(\tau_{1}^{\prime}, \tau_{2}^{\prime}, \tau_{3}^{\prime}\right)=\sqrt{\frac{1}{2\left|T_{\lambda}^{n}\right|}} \psi_{\ell}^{m}\left(\tau_{1}, \tau_{2}, \tau_{3}\right), \quad m=1,2,3, \quad \ell=1, \ldots, N_{k} .
$$

In [7], a similar multiwavelet basis is constructed, but normalization is done in the $L^{\infty}$-norm instead of the $L^{2}$-norm.

\subsection{Multiwavelet decomposition}

In Section 3.2, the relation between the DG approximation and the scaling-function coefficients on level $n$ was given. In this section, the scaling-function expansion on level $n$ is decomposed to a multiwavelet expansion on level $n-1$ [21], using the same notation as in [17]. The full decomposition is derived in $[7,14,15]$.

In the following, the scaling-function basis of $\mathbb{P}^{k}\left(T_{\lambda}^{n-1}\right)$ is written in terms of a vector $\boldsymbol{\phi}_{\lambda}^{n-1}=\left(\phi_{1 \lambda}^{n-1}, \ldots, \phi_{N_{k} \lambda}^{n-1}\right)^{\top}$. Because $V_{n-1}^{k+1} \subset V_{n}^{k+1}$, we can express $\boldsymbol{\phi}_{\lambda}^{n-1}$ in terms of $\boldsymbol{\phi}_{\lambda_{i}}^{n}, i=0,1,2,3$, using the local numbering $T_{\lambda}^{n-1}=T_{\lambda_{0}}^{n} \cup T_{\lambda_{1}}^{n} \cup T_{\lambda_{2}}^{n} \cup T_{\lambda_{3}}^{n}$ (Figure 2). This means that

$$
\boldsymbol{\phi}_{\lambda}^{n-1}=H_{0} \boldsymbol{\phi}_{\lambda_{0}}^{n}+H_{1} \boldsymbol{\phi}_{\lambda_{1}}^{n}+H_{2} \boldsymbol{\phi}_{\lambda_{2}}^{n}+H_{3} \boldsymbol{\phi}_{\lambda_{3}}^{n} .
$$

The $N_{k} \times N_{k}$ matrices $H_{i}$ are similar to the QMF coefficients in the one-dimensional case $[16,17]$, and are defined as $\left(i=0,1,2,3, p, q=1, \ldots, N_{k}\right)$

$$
\left(H_{i}\right)_{p, q}=\left\langle\phi_{p \lambda}^{n-1}, \phi_{q \lambda_{i}}^{n}\right\rangle=\iint_{T_{\lambda_{i}}^{n}} \phi_{p \lambda}^{n-1}(x, y) \phi_{q \lambda_{i}}^{n}(x, y) d x d y,
$$

using that $\phi_{q \lambda_{i}}^{n}$ is only nonzero in $T_{\lambda_{i}}^{n}$. We transform to barycentric coordinates $\tau$ based on the vertices of $T_{\lambda_{i}}^{n}$. Using equations (1), (2) and (4), this yields

$$
\begin{aligned}
\left(H_{i}\right)_{p, q} & =2\left|T_{\lambda_{i}}^{n}\right| \sqrt{\frac{1}{2\left|T_{\lambda}^{n-1}\right|}} \sqrt{\frac{1}{2\left|T_{\lambda_{i}}^{n}\right|}} \int_{0}^{1} \int_{0}^{1-\tau_{1}} \phi_{p}\left(M_{T_{\lambda_{i}}^{n} \rightarrow T_{\lambda}^{n-1}} \tau\right) \phi_{q}(\tau) d \tau_{2} d \tau_{1} \\
& =\sqrt{\frac{\left|T_{\lambda_{i}}^{n}\right|}{\left|T_{\lambda}^{n-1}\right|} \int_{0}^{1} \int_{0}^{1-\tau_{1}} \phi_{p}\left(M_{T_{\lambda_{i}}^{n} \rightarrow T_{\lambda}^{n-1}} \tau\right) \phi_{q}(\tau) d \tau_{2} d \tau_{1}} \\
& =\frac{1}{2} \int_{0}^{1} \int_{0}^{1-\tau_{1}} \phi_{p}\left(M_{T_{\lambda_{i}}^{n} \rightarrow T_{\lambda}^{n-1}} \tau\right) \phi_{q}(\tau) d \tau_{2} d \tau_{1}
\end{aligned}
$$


since $\left|T_{\lambda_{i}}^{n}\right|=\left|T_{\lambda}^{n-1}\right| / 4$. For a structured triangular mesh, the matrices $H_{i}$ do not depend on the mesh size [15].

Similarly, the multiwavelet basis is written as $\psi_{\lambda}^{m, n-1}=\left(\psi_{1 \lambda}^{m, n-1}, \ldots, \psi_{N_{k} \lambda}^{m, n-1}\right)^{\top}$, $m=1,2,3$. Because $W_{n-1}^{k+1} \subset V_{n}^{k+1}$, the vectors of multiwavelets can be written as

$$
\boldsymbol{\psi}_{\lambda}^{m, n-1}=G_{m, 0} \boldsymbol{\phi}_{\lambda_{0}}^{n}+G_{m, 1} \boldsymbol{\phi}_{\lambda_{1}}^{n}+G_{m, 2} \boldsymbol{\phi}_{\lambda_{2}}^{n}+G_{m, 3} \boldsymbol{\phi}_{\lambda_{3}}^{n}, \quad \text { for } m=1,2,3,
$$

with $\left(G_{m, i}\right)_{p, q}=\left\langle\psi_{p \lambda}^{m, n-1}, \phi_{q \lambda_{i}}^{n}\right\rangle, i=0,1,2,3, p, q=1, \ldots, N_{k}$. The matrices $G_{m, i}$ are computed similarly to the matrices $H_{i}$.

From equation (5) and the fact that $\mathbf{s}_{\lambda}^{n-1}=\left\langle f, \boldsymbol{\phi}_{\lambda}^{n-1}\right\rangle, \mathbf{d}_{\lambda}^{m, n-1}=\left\langle f, \boldsymbol{\psi}_{\lambda}^{m, n-1}\right\rangle$, it follows that we can decompose the scaling-function coefficients on level $n$ to scalingfunction and multiwavelet coefficients on level $n-1$ as follows:

$$
\begin{aligned}
& \mathbf{s}_{\lambda}^{n-1}=H_{0} \mathbf{s}_{\lambda_{0}}^{n}+H_{1} \mathbf{s}_{\lambda_{1}}^{n}+H_{2} \mathbf{s}_{\lambda_{2}}^{n}+H_{3} \mathbf{s}_{\lambda_{3}}^{n}, \\
& \mathbf{d}_{\lambda}^{1, n-1}=G_{1,0} \mathbf{s}_{\lambda_{0}}^{n}+G_{1,1} \mathbf{s}_{\lambda_{1}}^{n}+G_{1,2} \mathbf{s}_{\lambda_{2}}^{n}+G_{1,3} \mathbf{s}_{\lambda_{3}}^{n}, \\
& \mathbf{d}_{\lambda}^{2, n-1}=G_{2,0} \mathbf{s}_{\lambda_{0}}^{n}+G_{2,1} \mathbf{s}_{\lambda_{1}}^{n}+G_{2,2} \mathbf{s}_{\lambda_{2}}^{n}+G_{2,3} \mathbf{s}_{\lambda_{3},}^{n}, \\
& \mathbf{d}_{\lambda}^{3, n-1}=G_{3,0} \mathbf{s}_{\lambda_{0}}^{n}+G_{3,1} \mathbf{s}_{\lambda_{1}}^{n}+G_{3,2} \mathbf{s}_{\lambda_{2}}^{n}+G_{3,3} \mathbf{s}_{\lambda_{3}}^{n},
\end{aligned}
$$

which is called the multiwavelet decomposition from level $n$ to level $n-1$.

\section{Multiwavelet troubled-cell indicator}

In this section, multiwavelet troubled-cell indicators are defined for triangular meshes [17]. Here, the number of multiwavelet coefficients is increased by a renumbering technique $[17,19]$. This leads to the multiwavelet coefficients $\tilde{d}_{\ell \lambda}^{m, n-1}$, where $\ell=1, \ldots, N_{k}, m=1,2,3$, and $\lambda$ belongs to the triangles in level $n$ (instead of level $n-1)$.

\subsection{Parameter-based indicator}

The parameter-based multiwavelet troubled-cell indicator is defined similarly to the indicator for the one-dimensional and tensor-product two-dimensional case [17, 19]. The major difference lies in the number of coefficients that is needed for accurate detection. In the one-dimensional or tensor-product two-dimensional case, knowledge of the jump relation at element boundaries made it possible to use one coefficient per element for detection. In the triangular case, however, such a relation has not yet been proven, neither theoretically, nor numerically. Therefore, we will use all multiwavelet coefficients for detection: triangle $T_{\lambda}^{n}$ is detected as troubled if for any $m=1,2,3, \ell=1, \ldots, N_{k}$ : 


$$
\left|\tilde{d}_{\ell \lambda}^{m, n-1}\right|>C \cdot \max _{T_{\lambda}^{n} \in \mathscr{T}^{n}}\left\{\left|\tilde{d}_{\ell \lambda}^{m, n-1}\right|\right\}
$$

where $C \in[0,1]$ is a parameter that defines the strictness of the indicator. The parameter $C$ is problem-dependent: it depends on the strength of different shocks in the domain. This limits the applicability of this troubled-cell indicator. Therefore, an outlier-detection approach is also considered.

\subsection{Outlier-detection approach}

In this section, a troubled-cell indication technique for the multiwavelet coefficients on a structured triangular mesh is proposed that is based on outlier detection. In this way, a problem-dependent parameter is not needed.

A triangle is detected as troubled if it is detected in either the $x$ - or the $y$-direction, using the one-dimensional approach [20]. Regions with triangles in the $x$-direction are split into local regions of size 16, as is visualized in Figure 3, and a similar approach is followed for regions in the $y$-direction. The resulting outlier-detection approach is given in Algorithm 1. Note that this approach is closely related to the outlier-detection algorithm for a rectangular tensor-product mesh [20].

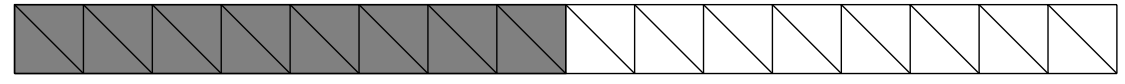

Fig. 3 Split of a 32-triangle region in the $x$-direction into two local regions of size 16 .

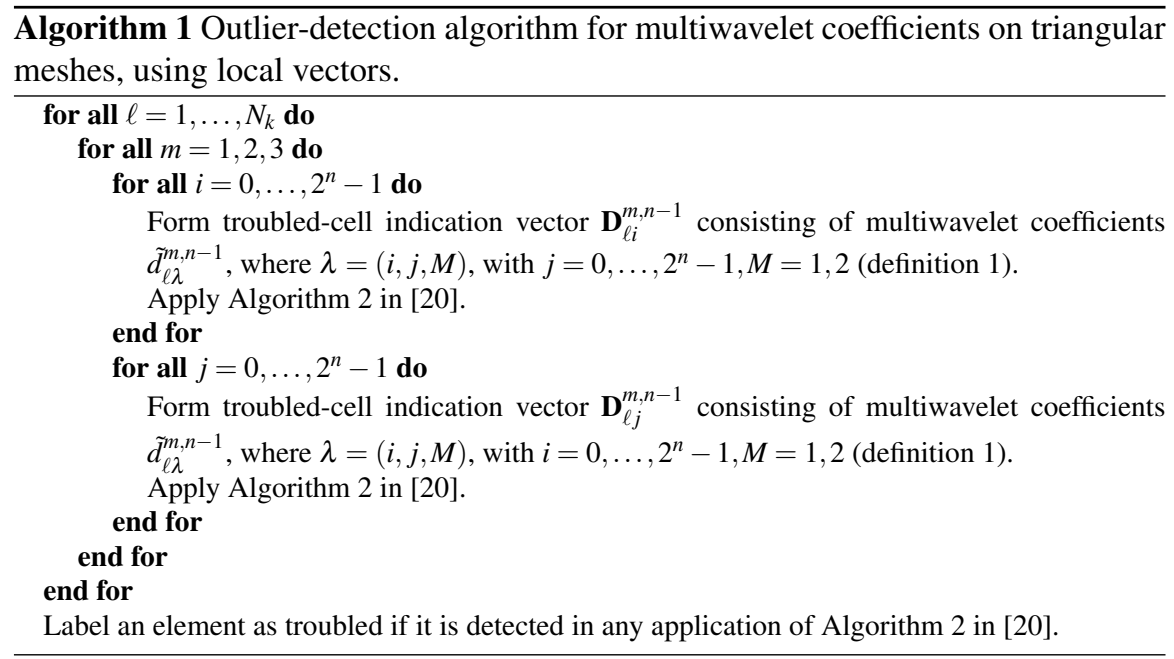




\section{Numerical results}

In this section, preliminary numerical results are shown for which the multiwavelet troubled-cell indicator has been tested [17]. The test is done for an example based on the linear advection equation on $[0,1] \times[0,1]$, given by $u_{t}+\nabla \cdot(\mathbf{v} u)=0$. Here, $\mathbf{v}=\left(v_{1}, v_{2}\right)^{\top}$ is the velocity vector, and $u=u(x, y, t)$ is the unknown quantity to be resolved. We use a diagonally-directed velocity: $\mathbf{v}=\sqrt{2} / 2 \cdot(1,1)^{\top}$. The following discontinuous initial condition is used:

$$
u_{0}(x, y)=\left\{\begin{array}{l}
1, \text { if }(x-0.5)^{2}+(y-0.5)^{2} \leq 0.1 \\
0, \text { else }
\end{array}\right.
$$

together with periodic boundary conditions. The exact solution of this boundaryvalue problem is equal to $u(x, y, t)=u_{0}\left(x-v_{1} t, y-v_{2} t\right)$. This means that the initial function should be recovered at the final time $T=\sqrt{2}$ [11].

The multiwavelet troubled-cell indicator is applied both using the parameter $C$ and with the outlier-detection approach. For the tests, the Matlab code of Hesthaven and Warburton is used [8], which is extended to the advection equation together with the vertex-based limiter by Raees et al. [13].

This section only shows the results for one specific initial condition. For more different test problems, we refer to [17].

\subsection{Multiwavelet coefficients of initial condition}

In order to investigate the information gleaned from multiwavelets on structured triangular meshes, in this section we first study multiwavelet coefficients of the initial condition in a DG basis (Figure 4). Clearly, the multiwavelet coefficients can be used to distinguish between smooth and nonsmooth regions. However, a clear meaning of the coefficients (as is the case in one and two dimensions) is difficult to establish.

\subsection{Detection at final time}

In this section, the approximations and detected troubled-cells are shown at the final time $T=\sqrt{2}$. Note that the exact solution equals the initial condition at this time.

The multiwavelet troubled-cell indicator is applied using either the parameterbased method or the outlier-detection approach (for which a problem-dependent parameter is not necessary). For the parameter-based method, the vertex-based limiter is applied only in the detected elements [10]. The outlier-detection scheme, however, turned out to only be stable if the limiter is also applied to all boundary elements. These elements are not always detected by the outlier scheme and are therefore not 


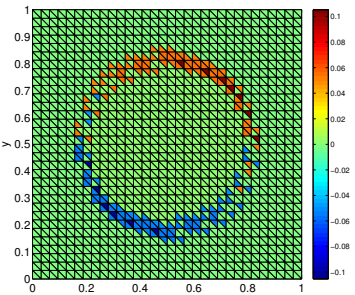

(a) $d_{1 \lambda}^{1, n-1}$

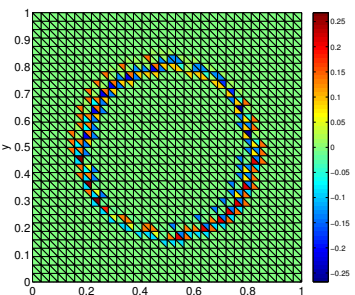

(d) $d_{1 \lambda}^{2, n-1}$

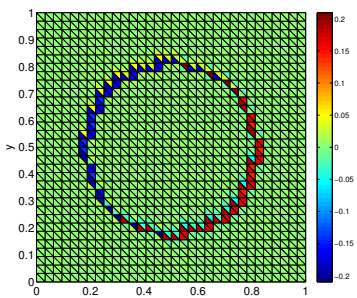

(g) $d_{1 \lambda}^{3, n-1}$

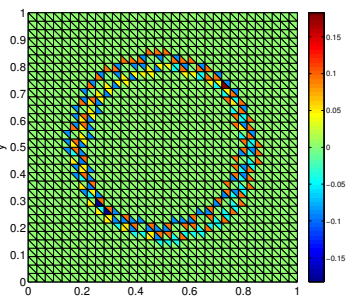

(b) $d_{2 \lambda}^{1, n-1}$

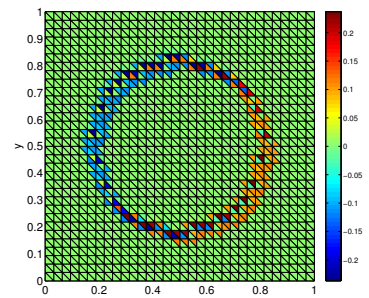

(e) $d_{2 \lambda}^{2, n-1}$

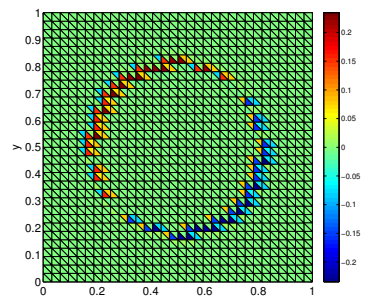

(h) $d_{2 \lambda}^{3, n-1}$

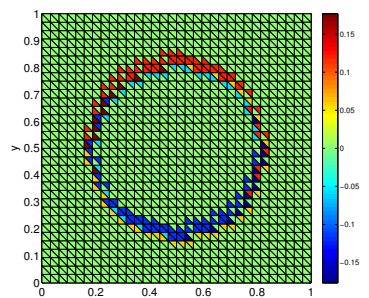

(c) $d_{3 \lambda}^{1, n-1}$

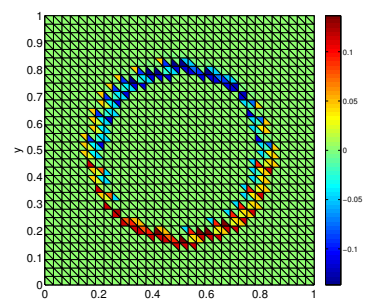

(f) $d_{3 \lambda}^{2, n-1}$

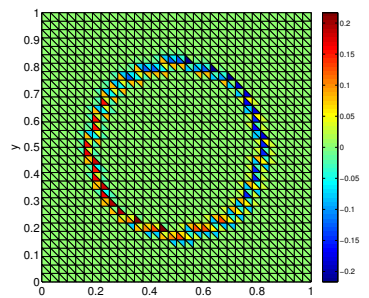

(i) $d_{3 \lambda}^{3, n-1}$

Fig. 4 Multiwavelet coefficients of initial condition, structured triangular mesh based on $32 \times 32$ rectangles, $k=1$.

marked as such in the figures. Similar boundary problems were also observed in [6], where it was proposed to either use an adaptive mesh with more triangles near the boundary or ignore the boundary triangles for certain resolution levels.

The results are shown in Figure 5. The parameter-based method detects the discontinuities accurately if a suitable value for the parameter $C$ is chosen. The outlierdetection method detects more elements near the circle wave but is not as sharp as we expect compared to results for the quadrilateral mesh case [20]. Inspection of the multiwavelet coefficients at the final time reveals that the discontinuous region is spread out wide, and therefore, the local region of size 16 is too small to contain both continuous and discontinuous regions. At certain locations, all coefficients in a local vector belong to a discontinuous region, and therefore, the fences are wide 
enough such that no elements are detected. Further research is needed to understand which outlier-detection strategy should be used.

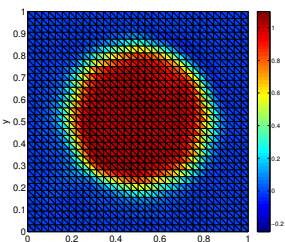

(a) $C=0.9$

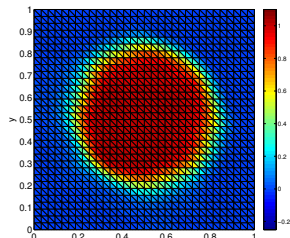

(b) Outlier

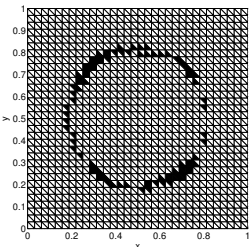

(c) $C=0.9$

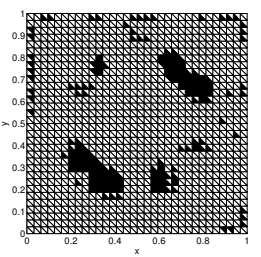

(d) Outlier

Fig. 5 Final-time approximations and corresponding detected troubled cells, using the parameterbased multiwavelet troubled-cell indicator or outlier detection on the multiwavelet coefficients, $T=\sqrt{2}$, structured triangular mesh based on $32 \times 32$ rectangles, $k=1$.

\section{Conclusion}

In this paper, the use of multiwavelets for troubled-cell indication on structured triangular meshes has been investigated. Inspection of the multiwavelet coefficients reveals that they are very useful to detect nonsmooth regions in the underlying function. Two different troubled-cell indicators were introduced: one indicator that uses a problem-depending parameter, and another indicator that applies outlier detection to the multiwavelet coefficients. By using outlier detection, a problem-dependent parameter is no longer needed.

Preliminary results have been shown for a test based on the two-dimensional linear advection equation. The parameter-based troubled-cell indicator detects the correct features if a suitable choice for the parameter is made. For the outlier-detection method, it seems as if the optimal size of the local vectors is no longer equal to 16.

More research should be done to recognize which multiwavelet coefficient measures which feature of the underlying function. Also, an improvement of the outlierdetection strategy is needed to detect the correct regions after time integration. Furthermore, tests for nonlinear PDEs such as the two-dimensional Euler equations, and comparisons with the KXRCF shock detector and the minmod-based TVB indicator should be performed to thoroughly test the applicability of multiwavelets and outlier detection for troubled-cell indication on triangular meshes. 


\section{References}

1. B.K. Alpert. A Class of Bases in $L^{2}$ for the Sparse Representation of Integral Operators. SIAM Journal on Mathematical Analysis, 24(1):246-262, 1993.

2. B. Cockburn. An Introduction to the Discontinuous Galerkin Method for ConvectionDominated Problems. In Advanced Numerical Approximation of Nonlinear Hyperbolic Equations, volume 1697 of Lecture Notes in Mathematics, pages 151-268. Springer Berlin Heidelberg, 1998.

3. B. Cockburn and C.-W. Shu. The Runge-Kutta Discontinuous Galerkin Method for Conservation Laws V: Multidimensional Systems. Journal of Computational Physics, 141(2):199-224, 1998.

4. M. Dubiner. Spectral methods on triangles and other domains. Journal of Scientific Computing, 6(4):345-390, 1991.

5. M.A. Fortes and M. Moncayo. Multiresolution analysis and supercompact multiwavelets for surfaces. Mathematics and Computers in Simulation, 81(10):2129-2149, 2011.

6. M.A. Fortes and M.L. Rodríguez. Non-uniform multiresolution analysis for surfaces and applications. Applied Numerical Mathematics, 75:123-135, 2014.

7. N. Gerhard and S. Müller. Adaptive multiresolution discontinuous Galerkin schemes for conservation laws: multi-dimensional case. Computational and Applied Mathematics, 35:321349, 2016.

8. J.S. Hesthaven and T. Warburton. Nodal Discontinuous Galerkin Methods: Algorithms, Analysis, and Applications, volume 54 of Texts in Applied Mathematics. Springer, New York, 2008.

9. T. Koornwinder. Two-Variable Analogues of the Classical Orthogonal Polynomials. In R. Askey, editor, Theory and Application of Special Functions, pages 435-495. Elsevier BV, 1975.

10. D. Kuzmin. A vertex-based hierarchical slope limiter for $p$-adaptive discontinuous Galerkin methods. Journal of Computational and Applied Mathematics, 233(12):3077-3085, 2010.

11. R.J. LeVeque. Finite Volume Methods for Hyperbolic Problems. Cambridge Texts in Applied Mathematics. Cambridge University Press, New York, sixth edition, 2002.

12. A. Meister, S. Ortleb, T. Sonar, and M. Wirz. An extended Discontinuous Galerkin and Spectral Difference Method with modal filtering. ZAMM - Journal of Applied Mathematics and Mechanics / Zeitschrift für Angewandte Mathematik und Mechanik, 93(6-7):459-464, 2013.

13. F. Raees, D.R. van der Heul, and C. Vuik. A mass-conserving level-set method for simulation of multiphase flow in geometrically complicated domains. International Journal for Numerical Methods in Fluids, 81(7):399-425, 2016.

14. A.B. Shelton. A multi-resolution discontinuous Galerkin method for unsteady compressible flows. PhD thesis, Georgia Institute of Technology, 2008.

15. F. Sieglar. Konstruktion von Multiwavelets auf Dreiecksgittern. Bachelor's thesis, RheinischWestfälischen Technischen Hochschule Aachen, 2013.

16. M.J. Vuik. Limiting and shock detection for discontinuous Galerkin solutions using multiwavelets. Master's thesis, Delft University of Technology, 2012.

17. M.J. Vuik. The use of multiwavelets and outlier detection for troubled-cell indication in discontinuous Galerkin methods. PhD thesis, Delft University of Technology, 2017.

18. M.J. Vuik and J.K. Ryan. Multiwavelet troubled-cell indicator for discontinuity detection of discontinuous Galerkin schemes. Journal of Computational Physics, 270:138 - 160, 2014.

19. M.J. Vuik and J.K. Ryan. Multiwavelets and Jumps in DG Approximations. In R.M. Kirby, M. Berzins, and J.S. Hesthaven, editors, Spectral and High Order Methods for Partial Differential Equations - ICOSAHOM 2014, volume 106 of Lecture Notes in Computational Science and Engineering. Springer International Publishing, 2015.

20. M.J. Vuik and J.K. Ryan. Automated parameters for troubled-cell indicators using outlier detection. SIAM Journal on Scientific Computing, 38(1):A84 - A104, 2016.

21. T.P.Y. Yu, K. Kolarov, and W. Lynch. Barysymmetric Multiwavelets on Triangle. Technical Report 1997-006, Interval Research Corporation, 1997. 